\title{
Cryo-Planing of Small Biological Specimens for SEM Using mPrep Capsules
}

\author{
William C. Plumley ${ }^{1}$ \\ 1. Electron Microscopy Program, Madison College, Madison, Wisconsin, USA
}

When preparing biological specimens for the scanning electron microscope (SEM), using a cryo technique often yields the best results. Specimen structure is often better preserved, and time and reagents are saved versus chemical fixation. With freeze fracturing, a common cryo technique, fractures tend to break along major features, rather than through them. Often, a sectioned view inside these features is desired, such as the interior of cells (figure 1). A way to achieve this is with cryo-planing: loading frozen samples into a cryo ultramicrotome and facing the sample with a glass knife, giving the sample a sectioned plane that makes for easy imaging in the SEM.[1]

However, this method has a major flaw; smaller $(<1 \mathrm{~cm})$ samples are difficult to handle without damage. But smaller means faster freezing and fewer artefacts, and for many specimens, like conifer needles or core biopsies, small is the only option. To get around this, and to add other benefits, a new procedure was developed, using mPrep/s capsules and workstation.[2] Besides the capsules, the materials and techniques used are common in an EM lab, keeping costs and training minimal.

mPrep, by Microscopy Innovations, is a specimen preparation system with two types of capsules: $\mathrm{mPrep} / \mathrm{s}$ for processing and sectioning, and $\mathrm{mPrep} / \mathrm{g}$ (not used here) for grid staining and storage. $\mathrm{mPrep} / \mathrm{s}$ features a removable screen with a slit that can pinch samples in place. The workstation holds the slit open, and aids in inserting the screen into the capsule. With the screen inserted, the capsules protect samples, and minimize direct handling of the sample, making damage less likely. The capsule also acts in place of a sample chuck, allowing easy preparation of multiple samples.

The $\mathrm{mPrep} / \mathrm{s}$ components did require slight modification. A surgical blade was used to increase the opening of the slits to fit the samples, making loading easy and keeping a firm hold on the sample, without applying too much pressure and risking breaking the sample. The capsules being closed at the sample end would require sectioning through the plastic, which is stiff at cryo temperatures. Trimming it $5 \mathrm{~mm}$ from the end exposed just the sample, made it easy to handle in the cryo chamber, and still gave enough taper in the capsule to hold the screen (figure 2).

Two specimens were processed in each run: Christmas cactus leaves, and canine kidney. The leaves were simply trimmed down, nothing more. The kidney was retrieved from Karnovsky's fixative, washed in sodium phosphate buffer, trimmed down, treated in $2 \%$ osmium tetroxide for an hour, and rinsed with water. While fresh kidney would've been preferable, it was not available. Optimal sample size seemed to be $1-1.5 \mathrm{~mm}$ in diameter, $5 \mathrm{~mm}$ in length. This maximized ease of loading, durability of sample, and viewable surface in the SEM.

The trimmed and treated samples were loaded into the screens, then into the capsules, and then submerged in liquid nitrogen until ready to be faced with pre-chilled glass knives. The samples were loaded into the cryo ultramicrotome, the chuck clamping down on the capsule's open end, and then faced until the exposed surface was smooth. Samples were then swapped out and placed back in liquid nitrogen, with knives also changed as needed. 
After the samples were faced, they were then placed in a freeze dryer. Once this was complete, the samples were ready for mounting. The screens were removed from the capsules and excess sample on the back side is trimmed flush. The screens are then mounted to the stub with carbon tape and colloidal graphite with the screen the only part handled, already holding the sample securely face-up. After a sputter-coating of gold-palladium, the samples were ready to be observed in the SEM.

This modified procedure enabled easier, faster preparation of multiple small samples, while still maintaining the excellent morphology and sectioned view on a variety of specimens that cryo-planing is best known for, with only a minimal amount of additional materials and time. [3]

\section{References:}

[1] Jaap Njisse, and Adriaan C. van Aelst, Scanning 21 (1999), p. 372-378

[2] Catalogue nos. S0812 and WS100, Microscopy Innovations, Marshfield, WI

[3] Thanks to Michael Kostrna (Madison College) for his teaching and support, and Steve Goodman (Microscopy Innovations) and Ed Calomeni (Ohio State University) for their encouragement.
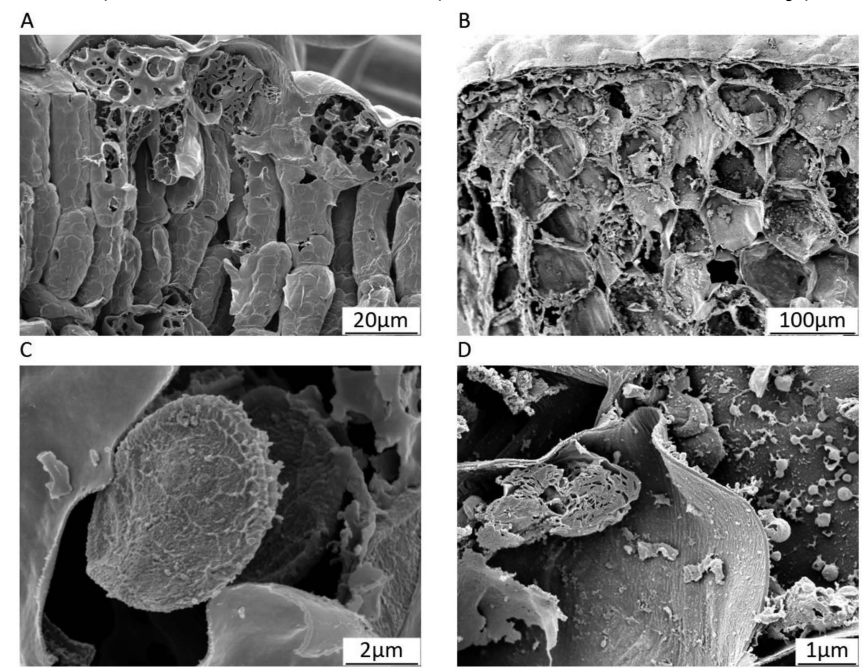

Figure 1. A: Freeze-fractured leaf with closed cells. B: Cryo-planed leaf with sectioned cells. C: Chloroplast in a freeze-fractured leaf. D: Sectioned chloroplast in a cryo-planed leaf.
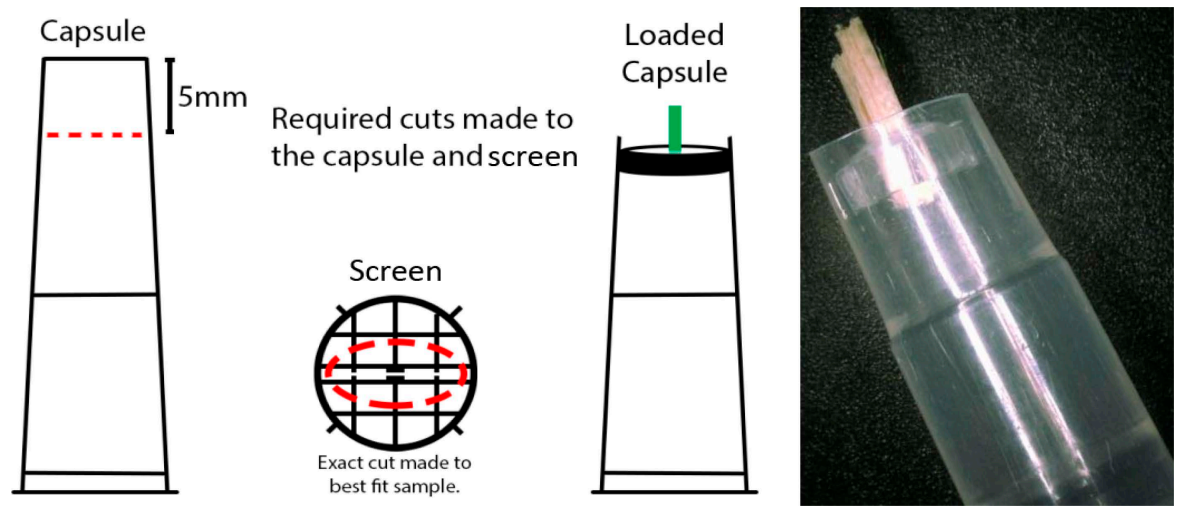

Figure 2. Capsule and screen modifications, and the capsule loaded with screen and sample. 